\title{
LncRNA MALAT1 exhibits positive effects on nucleus pulposus cell biology in vivo and in vitro by sponging miR-503
}

Hongyu Zheng ${ }^{1 \dagger}$, Tingting Wang ${ }^{2 \dagger}$, Xiangmin $\mathrm{Li}^{3}$, Wei He ${ }^{4}$, Zhiqiang Gong ${ }^{5}$, Zhenkai Lou ${ }^{5}$, Bing Wang ${ }^{5}$ and Xingguo Li $\mathrm{i}^{*}$

\begin{abstract}
Background: Intervertebral disc degeneration (IDD) is characterized by the loss of nucleus pulposus cells (NPCs) and phenotypic abnormalities. Accumulating evidence suggests that long noncoding RNAs (IncRNAs) are involved in the pathogenesis of IDD. In this study, we aimed to investigate the functional effects of IncRNA MALAT1 on NPCs in IDD and the possible mechanism governing these effects.

Results: We validated the decreased expression of MALAT1 in the IDD tissues, which was associated with decreased Collagen II and Aggrecan expression. In vitro, overexpressed MALAT1 could attenuate the effect of IL-1 $\beta$ on NPC proliferation, apoptosis, and Aggrecan degradation. In vivo, MALAT1 overexpression attenuated the severity of disc degeneration in IDD model rats. Our molecular study further demonstrated that MALAT1 could sponge miR503, modulate the expression of miR-503, and activate downstream MAPK signaling pathways. The effects of MALAT1 on NPCs were partially reversed/aggregated by miR-503 mimics/inhibitor treatment.

Conclusion: Our data suggested that the MALAT1-miR-503-MAPK pathway plays a critical role in NPCs, which may be a potential strategy for alleviating IDD.

Keywords: Intervertebral disc degeneration, Long noncoding RNA MALAT1, microRNA-503, Nucleus pulposus cells, Apoptosis, MAPK pathway
\end{abstract}

\section{Background}

Intervertebral disc degeneration (IDD) has been widely regarded as making a significant contribution to low back pain (LBP), a leading cause of chronic pain, at various times and is an important cause of a series of spinal degenerative diseases [1]. The intervertebral disc (IVD), consists of three structurally connected parts: the peripheral annulus fibrosus (AF), the central gelatinous nucleus pulposus (NP) and the cartilage endplates (CEPs) [2], and it is the largest avascular organ. Nucleus pulposus cells (NPCs)

\footnotetext{
* Correspondence: XingguoLi@tom.com

${ }^{\dagger}$ Hongyu Zheng and Tingting Wang contributed equally to this work. ${ }^{5}$ Department of Orthopedics, First Affiliated Hospital of Kunming Medical University, No. 295 Xichang Road, Kunming, Yunnan, China Full list of author information is available at the end of the article
}

are highly hydrated in healthy IVDs, which can produce abundant Aggrecan and Collagen II [3] and can ensure the IVD mechanical function of distributing the axial compressive forces acting on the spine and the multiaxial flexibility together with AF, cartilaginous and bony endplates [4]. Loss of NPCs [5, 6] and imbalance of matrix synthesis and degradation [7], play important roles in the occurrence and development of IDD. Therefore, targeting the function of NPCs represents a potential strategy for the improvement of IDD.

Long noncoding RNAs (lncRNAs) are a class of noncoding RNAs with a transcriptional length of more than 200 nucleotides, regulating gene expression in epigenetics, transcription, and post-transcription [8]. Recently, accumulating

C C The Author(s). 2020 Open Access This article is licensed under a Creative Commons Attribution 4.0 International License, which permits use, sharing, adaptation, distribution and reproduction in any medium or format, as long as you give appropriate credit to the original author(s) and the source, provide a link to the Creative Commons licence, and indicate if changes were made. The images or other third party material in this article are included in the article's Creative Commons licence, unless indicated otherwise in a credit line to the material. If material is not included in the article's Creative Commons licence and your intended use is not permitted by statutory regulation or exceeds the permitted use, you will need to obtain permission directly from the copyright holder. To view a copy of this licence, visit http://creativecommons.org/licenses/by/4.0/ The Creative Commons Public Domain Dedication waiver (http://creativecommons.org/publicdomain/zero/1.0/) applies to the data made available in this article, unless otherwise stated in a credit line to the data. 
evidence has shown that aberrantly expressed lncRNAs play a vital role in the IDD process. The levels of MALAT1 were significantly reduced in NPCs from IDD patients [9]. Recent reports found that MALAT1, metastasis-associated lung adenocarcinoma transcript-1, promoted caspase 3 activity, regulated the secretion of cytokines, and was involved in cell proliferation, migration, and apoptosis [10, 11]. These findings suggested that MALAT1 might participate in IDD development by inducing NPCapoptosis and the secretion of pro-inflammatory cytokines. However, little is known about the role and mechanism of MALAT1 in IDD.

The genetic mechanisms of IncRNAs primarily include miRNAs sponges. LncRNAs could posttranscriptionally interact with miRNAs to serve as competing endogenous RNAs (ceRNAs), thereby repressing miRNA expression, and can inhibit translation or degradation of miRNA downstream targets. Yan et al. [12] demonstrated that MALAT1 could directly bind to miR-503 and modulate the expression of miR-503. miR-503, located on the chromosome $\mathrm{Xq} 26.3$, is an intragenic miRNA and belongs to the miR-16 family [12]. Prevailing evidence suggests that miR-503 exerts diverse biological functions, such as osteoblast proliferation and apoptosis, which are potentially amenable to therapeutic manipulation for clinical application [13]. Additionally, in several cell lines, MALAT1 could regulate downstream MAPK and activator protein-1 (AP1) signaling pathways, which play a critical role in intervertebral disc degeneration [14-16]. However, the impact of MALAT1 on the MAPK/AP1 pathway in NPC has not been determined.

In the present study, we found lower levels of MALAT1 expression in IDD tissues and an association with Collagen II/Aggrecan. We also analyzed the functional effects of MALAT1 overexpression, and miR-503 mimics/inhibitor on NPC proliferation, apoptosis, and ECM degradation in vitro and in vivo. In addition, we investigated the involvement of the MAPK/AP1 signaling pathway in this process.

\section{Results \\ Expression of MALAT1 in lumbar IDD tissues and the correlation with the prognosis of IDD}

To investigate the effect of lnc-MALAT1 in IDD, we examined its expression in tissue specimens, including 10 normal specimens and 37 lumbar IDD specimens. The results showed that MALAT1 expression was significantly down-regulated in the lumbar IDD specimens (Fig. 1a). We evaluated the progression of the above 37 IDDs according to the Pfirrmann grading (mild degeneration: $3-4,21$, severely degraded: $5-6,16)$. The MALAT1 level was lower in severely IDD specimens than in mild IDD specimens (Fig. 1b). IDD is characterized by decreased cell number, increased matrix degradation [17] and increased proinflammatory cytokine release [18]. Therefore, we examined the expression of
IL-1 $\beta$, Collagen II and Aggrecan in IDD specimens and normal specimens. Compared with normal specimens, IL-1 $\beta$ mRNA levels were significantly up-regulated in IDD specimens, while Collagen II and Aggrecan levels were significantly down-regulated (Fig. 1c-e). This result suggests that MALAT1 may play a potential role in IDD progression.

\section{IL-1 $\beta$ inhibits the function of NPCs and the expression of MALAT1}

Elevated levels of proinflammatory mediators increased Aggrecan and Collagen II degradation, and increased degradation of extracellular matrix (ECM) has been widely regarded as a significant contributor to intervertebral disc degeneration (IDD). After stimulation with IL-1 $\beta$, the proliferation and number of human NPCs were inhibited (Fig. 2a-b). Additionally, IL-1 $\beta$ reduced the expression of MALAT1 (Fig. 2c). We found that IL-1 $\beta$ can reduce the expression of Aggrecan and Collagen II (Fig. 2d-f).

\section{MALAT1 modulates IL-1 $\beta$-induced dysfunction of NPC proliferation, apoptosis, and ECM degradation in vitro} Next, the function of MALAT1 in IL- $1 \beta$-stimulated NPCs was evaluated by measuring Collagen II and Aggrecan protein levels, cell proliferation, and cell senescence in human NPCs (Fig. 3a). MALAT1 overexpression reversed the effect of IL-1 $\beta$ on NPC proliferation (Fig. 3b), NPC number (Fig. 3c), and cell apoptosis (Fig. 3d-e) induced by IL-1 $\beta$. Overexpression of MALAT1 reversed the inhibitory effect IL-1 $\beta$ on Collagen II and Aggrecan protein levels (Fig. 3f-h). These data indicate that MALAT1 overexpression partially reverses the effect of IL-1 $\beta$ on NPC.

\section{MALAT1 modulates IL-1 $\beta$-induced IDD and inhibits apoptosis in the rat model}

After 4 weeks of modeling, characterization of rat lumbar vertebral IDD caused by unbalanced dynamics and static forces was observed on X-ray films. After the injection of MALAT1 into the L4/L5 and L5/L6 discs of the rats, there was no significant difference between the two groups (Fig. 4a). At 4 weeks after injection, the MRI of the intervertebral disc in the OV-MALAT1 group showed stronger signal intensity than the control group (Fig. 4b).

The intervertebral disc AF of the OV-MALAT1 group showed normal structure or mild serpentine appearance, while the control group showed mild to moderate appearance, eventually showing a serious serpentine appearance, contour reversal, and rupture (Fig. 4c). At four weeks after injection, the histological score of the OvMALAT1 group was significantly lower than that of the OV-NC group $(P<0.05)$ (Fig. $4 \mathrm{~d})$. The apoptosis of intervertebral disc cells was detected by in situ TUNEL staining. After four weeks of injection, the ratio of 

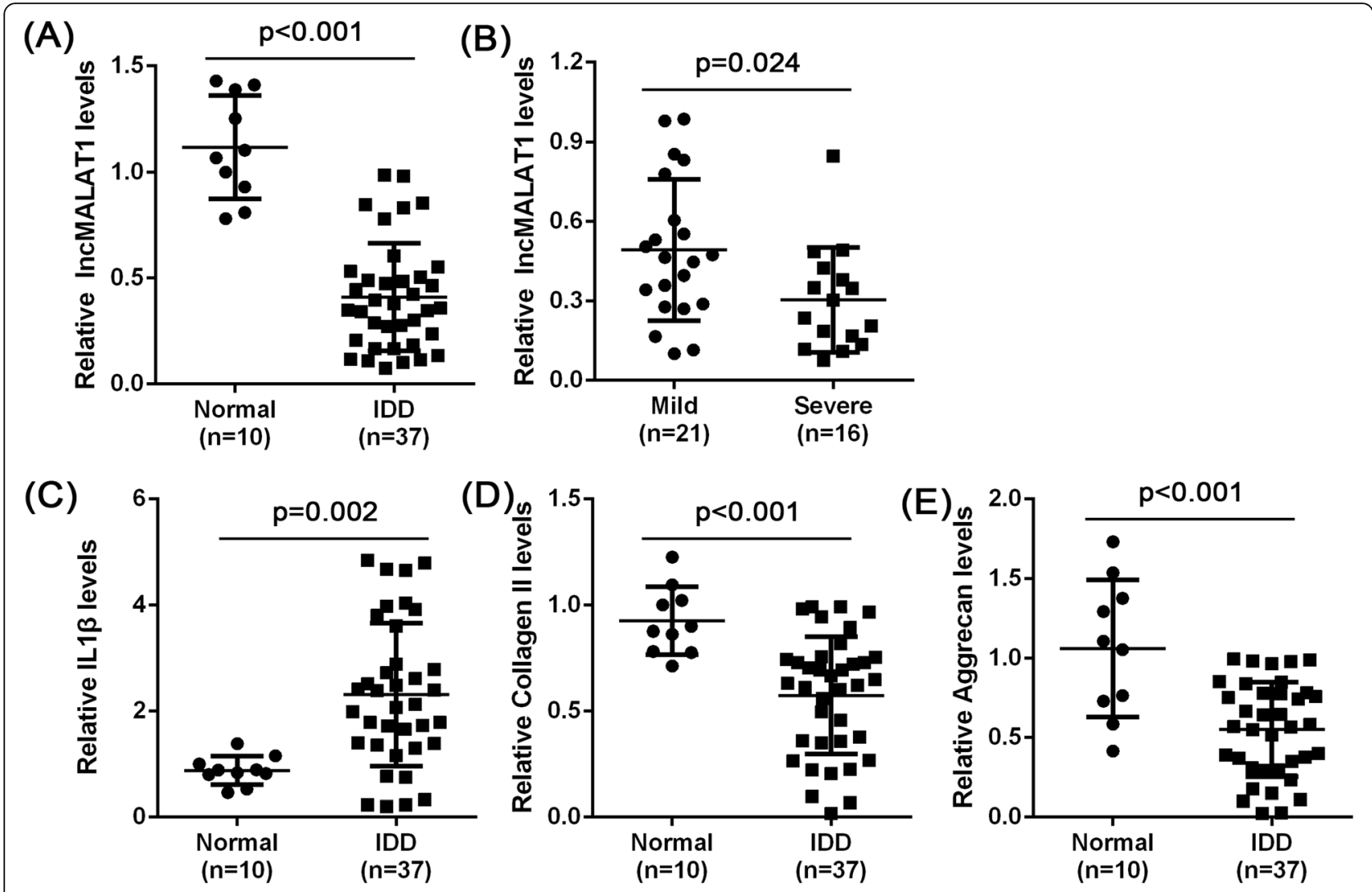

Fig. 1 The expression of MALAT1 in lumbar IDD tissues and the correlation with prognosis of IDD a MALAT1 expression in 10 normal and 37 IDD tissue specimens determined by real-time PCR assays. b MALAT1 expression in 37 IDD tissue specimens was delineated according to the Pfirrmann grading groups (mild $n=21$, severe $n=16$ ). $\mathbf{c}-\mathbf{e}$ The mRNA expression of IL-1 $\beta$, Collagen $\|$ and Aggrecan in 10 normal and 37 IDD tissue specimens was determined using real-time PCR assays. The data are presented as mean \pm SD of three independent experiments

apoptotic cells in the annulus fibrosus (external or internal) of the OV-MALAT1 group was significantly lower than that of the OV-NC group (Fig. 4e-f).

\section{MALAT1 acts as a sponge for miR-503}

In general, lncRNAs could interact with miRNAs, and affect miRNA-binding target genes and downstream signaling pathways. Therefore, we investigated whether miRNAs are involved in the effect of MALAT1 on IDD. Bioinformatic analysis showed that MALAT1 binds to miR-503 (Fig. 5a). Luciferase reporter experiments showed that the transfection of miR-503 mimics significantly inhibited the luciferase activity of wild-type MALAT1, but not mutated MALAT1 (Fig. 5b). Knockdown of MALAT1 significantly increased the level of miR-503 expression in NPCs (Fig. 5c), but the transfection of miR-503 mimics did not change the MALAT1 levels in NPCs (Fig. 5d). Additionally, the expression levels of miR-503 were detected in the clinical sample. As Fig. 5e shows, the miR-503 expression level was markedly higher in IDD tissue than in normal tissue. Furthermore, Spearman's rank correlation analysis revealed that MALAT1 expression was negatively correlated with miR-503 (Fig. 5f). These data indicated that MALAT1 acts as a sponge for miR-503.

MALAT1 inhibits miR-503 to regulate the function of NPCs and the MAPK pathway

We further verified the combined effect of MALAT1 and miR-503 on NPC proliferation, apoptosis, and ECM degradation, as well as the involvement of the MAPK pathway. Upon IL-1 $\beta$ stimulation, MALAT1 overexpression remarkably enhanced the NPC proliferation and NPC number (Fig. 6a-b) and reduced NPC apoptosis (Fig. 6cd), but was attenuated by miR-503 mimics or aggravated by miR-503 inhibitor treatment. Furthermore, MALAT1 overexpression increased the protein levels of Collagen II and Aggrecan; the effect of MALAT1 overexpression was partially eliminated by miR-503 mimics. In contrast, miR503 inhibitor increased Collagen II and Aggrecan protein levels (Fig. 6e-g).

Previous studies have shown that MALAT1 can affect the MAPK/AP1 signaling pathway [19], which is an important pathway for IVD [15]. Therefore, we examined the relationship between MALAT1, miR-503 and the MAPK pathway. Immunoblot results showed that overexpression 


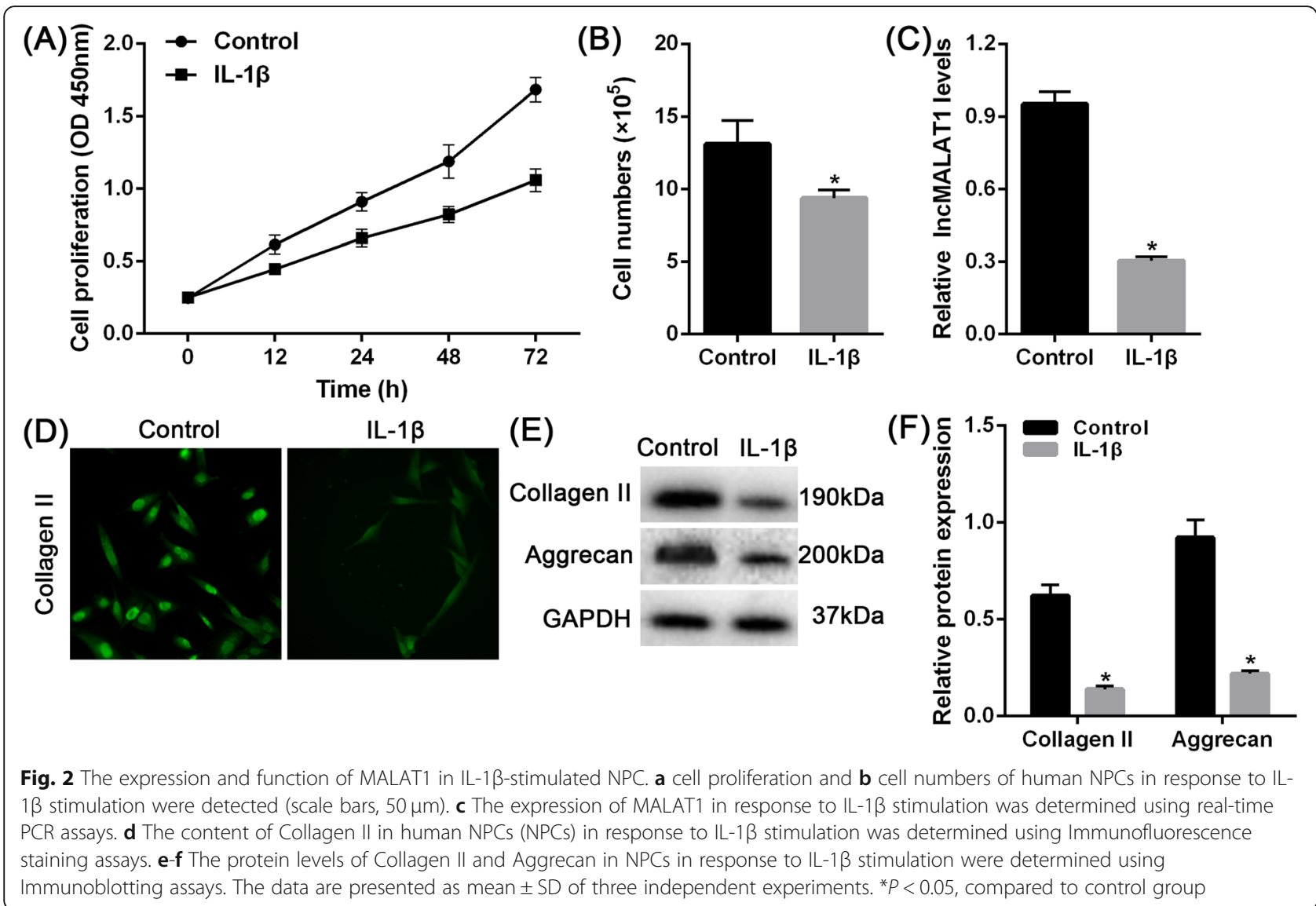

of MALAT1 significantly reduced IL-1 $\beta$-induced phosphorylation levels of p-fos, p-p38, and p-cJuN, and the effect of MALAT1 overexpression was partially eliminated by miR503 mimics or aggravated by a miR-503 inhibitor (Fig. 7).

\section{Discussion}

An increasing number of study have indicated the important roles of lncRNAs in IDD. In this study, we demonstrated that MALAT1 was downregulated in IDD tissues. MALAT1 overexpression promoted NPC proliferation and suppressed IL-1 $\beta$-induced apoptosis, as well as IL-1 $\beta$-induced degradation of ECM, indicating the protective effect of MALAT1 in IDD. Furthermore, we found that MALAT1 could sponge miR-503 and regulate the downstream factors of MAPAK pathway.

During the progression of IDD, NPCs produce excessive inflammatory mediators including TNF- $\alpha$ and IL-1 $\beta$ $[20,21]$, which is considered an important cause of IDD [22-24]. This phenomenon is observed with the senescence or apoptosis of NPCs, as well as the degradation of many protein components of the extracellular matrix (ECM) including Collagen II and Aggrecan. Therefore, we first focused on the expression of MALAT1 and ECMdegrading Collagen II/Aggrecan between normal and degenerative IVD tissues. The results showed that MALAT1 levels were decreased in IDD tissues, and Collagen II/ Aggrecan were decreased. Moreover, in severe degeneration specimens, MALAT1, Collagen II, and Aggrecan levels were more downregulated. In Zhang et al. 's study [25], the expression level of MALAT1 was significantly reduced in NP cells isolated from IDD patients compared with controls. These findings suggested that reduced MALAT1 expression might participate in IDD development.

It is well known that NPCs are involved in resisting mechanical loads, and the synthesis of ECM is important to maintain spinal stability. The loss of NPCs correlates with the pathological process of IDD [26]. Therefore, we next focused on the effect of MALAT1 overexpression on NPC apoptosis and ECM degradation. Functionally, restored expression of MALAT1 partially attenuated the IL-1 $\beta$ induced suppression of NPC proliferation, cell apoptosis, and degradation of ECM. At seven weeks after injection with a MALAT1-overexpressing lentiviral vector, rats appeared to have reduced spine curvature. Although no improved effect on the characterization of lumbar IDD was observed from X-ray, MALAT1 overexpression treatment improved the overall histological score shown by HE staining, which coincided with the degree of disc degeneration by MRI imaging. Under unbalanced dynamic and static forces of the spine, apoptosis 


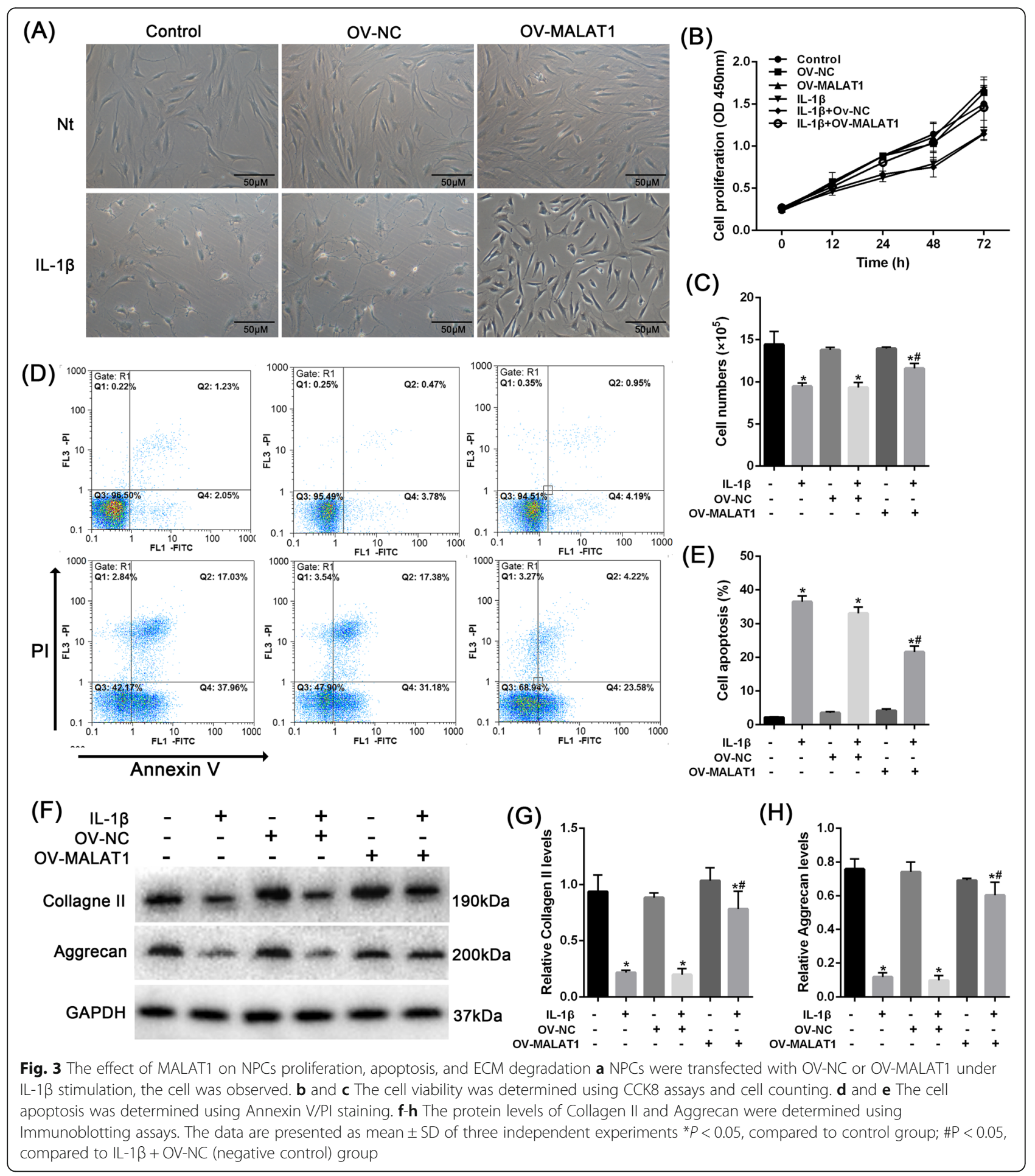

in disc cells could be induced in the rat model [27]. In vitro, MALAT1 overexpression reduced IL-1 $\beta$-induced apoptosis of NPCs. Additionally, we found that MALAT1 overexpression treatment could reduce TUNEL-positive NPCs in IDD rats. These findings indicate that suppressing NPC apoptosis by MALAT1 overexpression may contribute to the attenuation of IDD.
As mentioned, the primary mechanism of lncRNAs may serves as ceRNA by sponging miRNAs to regulate the downstream target mRNAs or related signal pathways. Based on a previous study and bioinformatic soft prediction, miR-503 might be a potential target of MALAT1 [12]. The luciferase reporter assay confirmed this prediction. Furthermore, miR-503 suppression mitigated the 


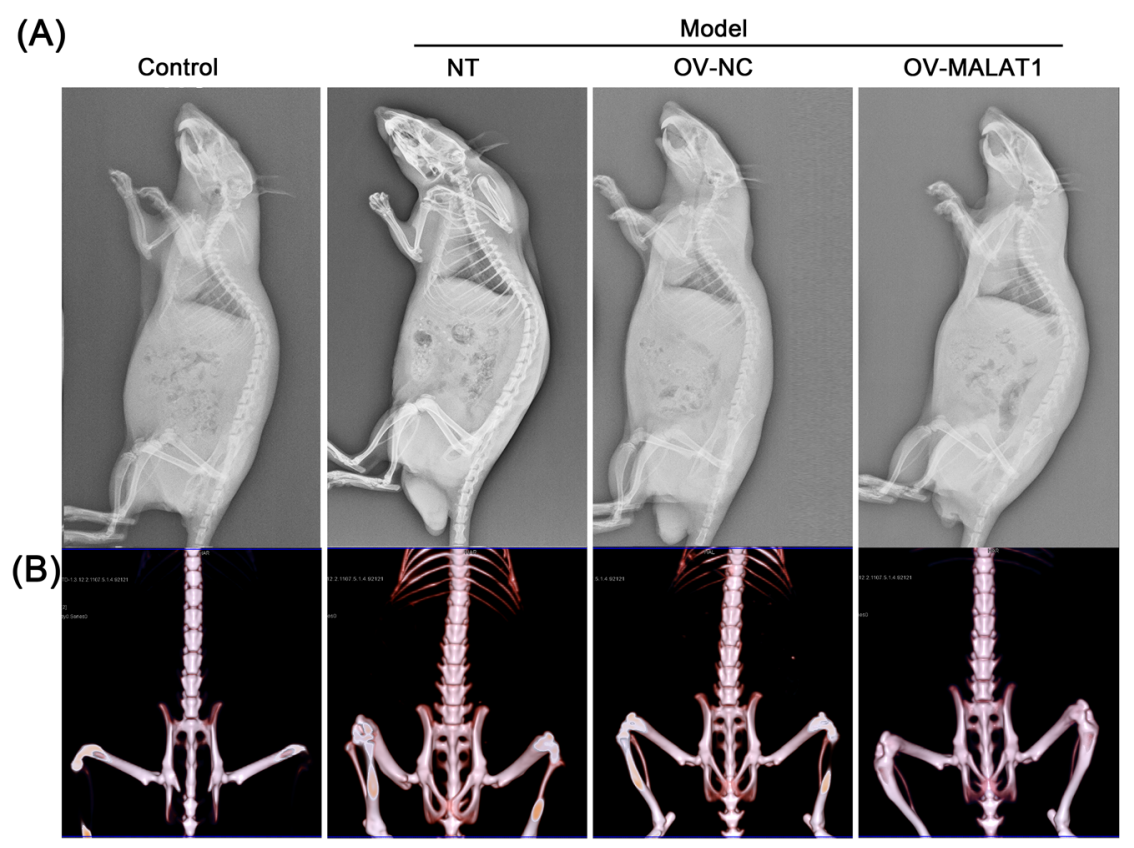

(C)

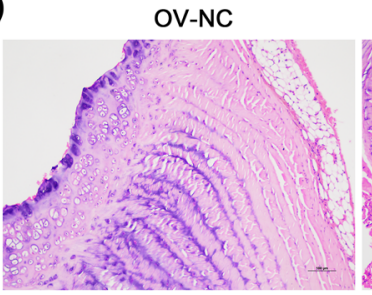

(E)

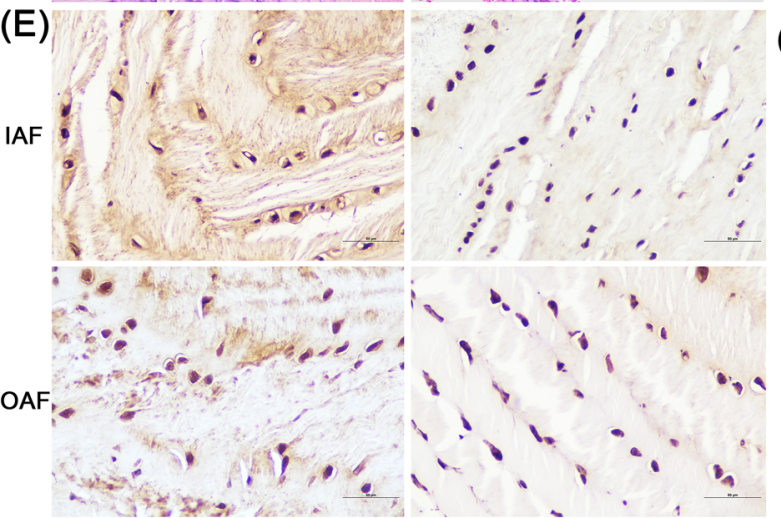

(D)

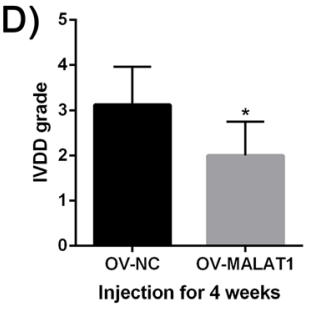

(F)

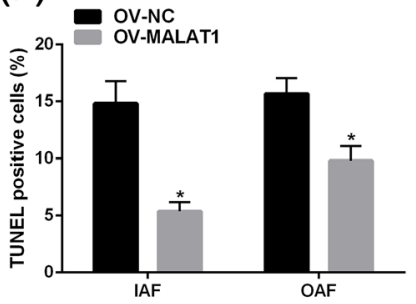

Injection for 4 weeks

Fig. 4 The function of MALAT1 in vivo (a) Lateral radiographies of the rat lumbar spine and disc height index (DHI) in control groups andMALAT1 group. b MRI at seven weeks after injection and change of MRI grade in control groups and MALAT1 group. c-d HE staining and comparison of the grade of IDD control groups and MALAT1 group. e-f Cell apoptosis according to in situ TUNEL staining. The data are presented as mean \pm SD of three independent experiments. ${ }^{*} P<0.05$, compared to OV-NC group

positive effects of MALAT1 on NPC cell proliferation and apoptosis induced by IL-1 $\beta$, while miR-503 mimics enhanced this effect. A previous study showed that miR-503 plays an important role in the pathogenesis of postmenopausal osteoporosis [28]. In IVD, both Ji et al. [29] and Li et al. [30]'s study demonstrated that miR-503 levels are significantly upregulated in IVD patients compared with controls, which is consistent with our results in this study.
Degeneration of the IVD appears to be mediated by several pathways. The MAPK pathway has been regarded as a crucial regulators of IVVD [31]. The MAPKs pathway can activate the downstream transcription factor AP1 (JUN, ATF, c-Fos, Maf) and then control many cellular processes including cell growth, differentiation, and apoptosis [32-34]. Several studes have shown that inhibition of MAPK/AP1 pathway is a potential 


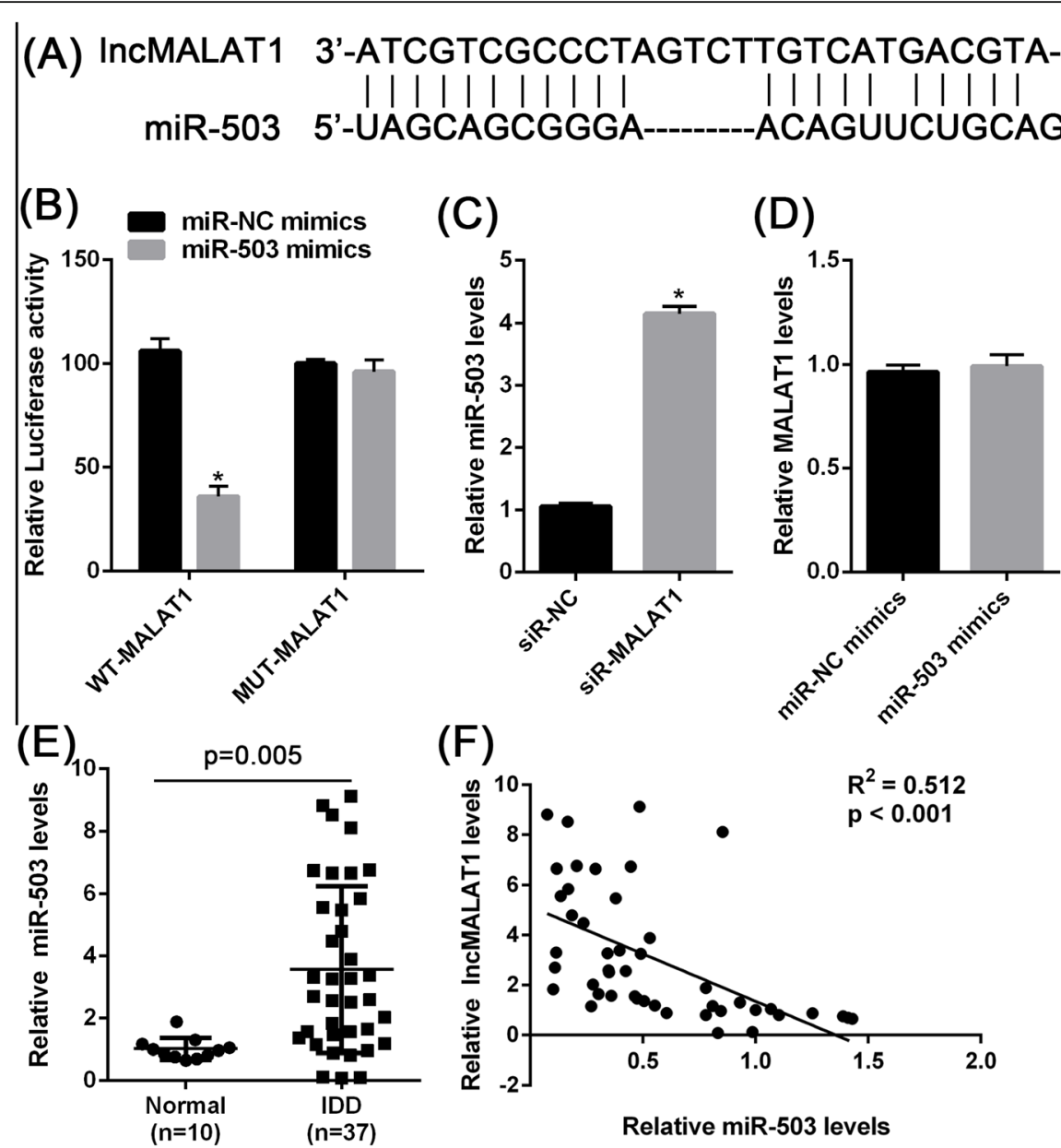

Fig. 5 MALAT1 acts as a sponge for miR-503 (a) Schematic representation of binding sites between MALAT1 and miR-503 predicted by StarBase software. $\mathbf{b}$ Wild-type and mutant-type MALAT1 luciferase reporter gene vectors were constructed and named wt-MALAT1 and mut-MALAT1; mut-MALAT1 contained a 4 bp mutation on any of the predicted miR-503 binding sites. The above vectors were co-transfected into HEK-293 cells with miR-NC or miR-503 mimics; the luciferase activity was determined. c NPCs were transfected with MALAT1 siRNA; the expression of miR-503 was determined using real-time PCR. $\mathbf{d}$ NPCs were transfected with miR-NC or miR-503 mimics; the expression of MALAT1 was determined using real-time PCR. e The expression levels of miR-503 in IDD tissues and normal ones were determined by RT-qPCR. $\mathbf{f}$ The correlation between MALAT1 and miR-503 was analyzed using Spearman's rank correlation analysis. The data are presented as mean \pm SD of three independent experiments. ${ }^{*} P<0.05$

therapeutic target for IDD. Our data showed that MALAT1 overexpression decreased IL-1 $\beta$-upregulated p38, JUN and Fos levels, and miR-503 mimics or inhibitors attenuated or enhanced their phosphorylation level. These results are also consistent with previous studies [35-37], and imply that the mechanism governing MALAT1 may be the MAPK/AP1 signaling pathway. In addition, we found that miR-503 can antagonize the regulation of MAPK by MALAT1. Unfortunately, the mechanism governing the activity of miR-503 in the MAPK/AP1 pathway was not investigated. According to the existing evidence, miR-503 may not directly target genes in the MAPK/AP1 pathway, but indirectly regulate the MAPK/AP1 pathway by targeting such proteins as
RANK and NFkB $[28,38]$. We also predicted the targets of miR-503 through miRwalk 3.0 software [39] and found a few putative targets in given pathways, including MAPK, Wnt, Insulin pathway et al. (Table S1). This finding supports the potential role of MAPK/AP1 pathways in IVD degeneration.

\section{Conclusions}

MALAT1 acts as a sponge and ceRNA for miR-503 and alleviates IL-1 $\beta$-induced NPC apoptosis and degenerative processes through the MAPK signaling pathway. Our present study may help to elucidate the molecular mechanisms underlying IDD and provides a potentially effective therapeutic strategy for IDD. 

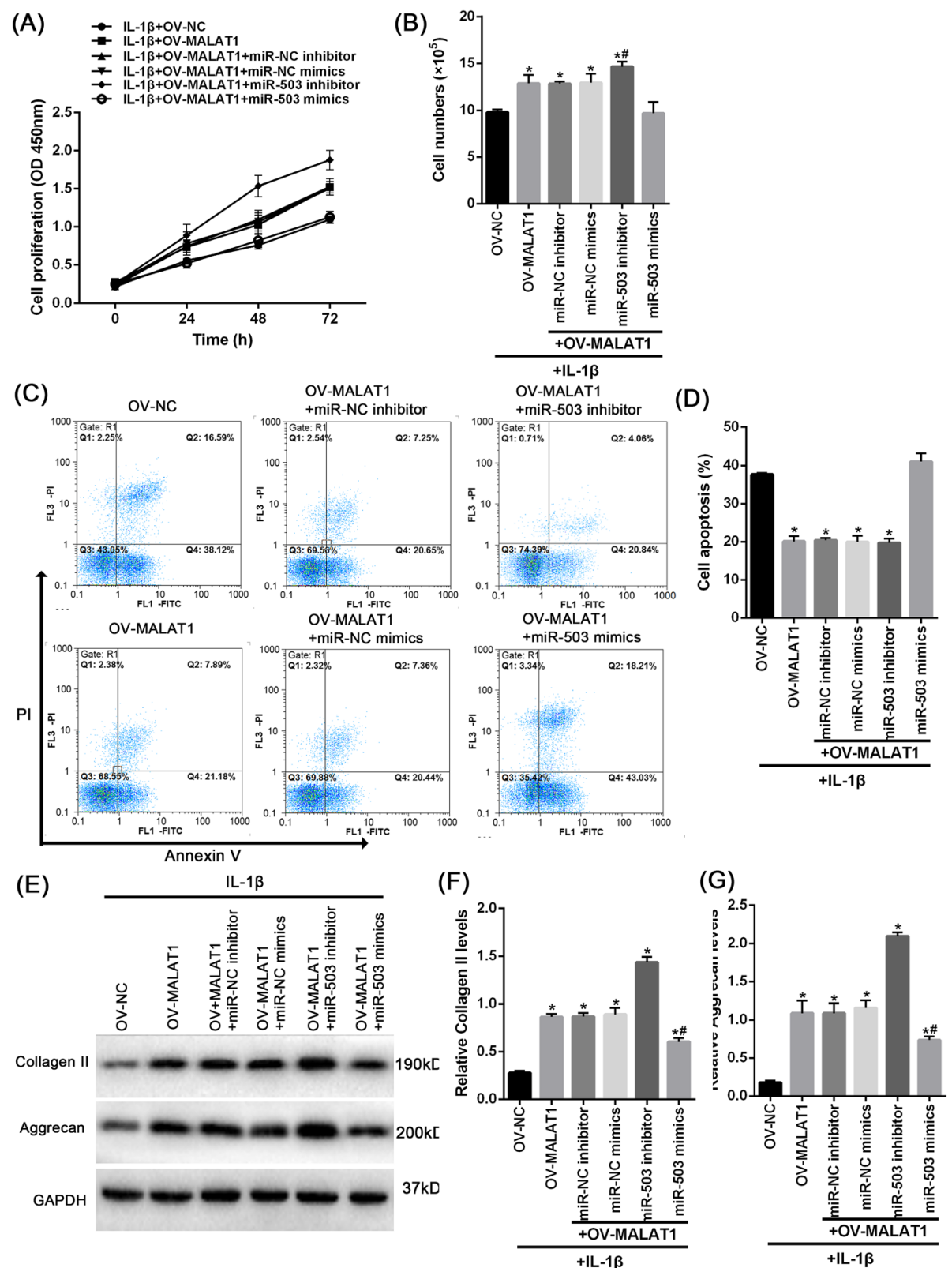

Fig. 6 the effect of miR-503 on MALAT1 modulate the function of NPC NPCs were co-transfected with miR-503 mimics or inhibitor and OVMALAT1 under IL-1 $\beta$ stimulation; (a) and (b) The cell viability was determined using CCK8 assays and cells counting. $\mathbf{c}-\mathbf{d}$ The cell apoptosis was determined using Annexin V/PI stain. e-g The protein levels of Collagen II and Aggrecan were determined using Immunoblotting assays. $\mathbf{d}$ The protein levels of the MAPK pathway were determined using Immunoblotting assays. The data are presented as mean \pm SD of three independent experiments. ${ }^{*} P<0.05$, compared to control group; \#\#P<0.01, compared to IL-1 $\beta+$ si-circSEMA4B group

\section{Methods}

\section{Human tissue samples}

This study was approved ethically by the First Affiliated Hospital of Kunming Medical University, and written informed consent was obtained from every participant. Degenerative NP tissues were obtained from 37 patients (average age $52.9 \pm 9.6$ ) with IDD, and normal NP tissues from 10 lumbar trauma patients (average age $21.7 \pm 3.2$ ) were excised by surgical resection, which was served as a control. The degree of disc degeneration was evaluated via a magnetic resonance imaging (MRI) scan according to the Pfirrmann grading classification. All specimens were collected within $3 \mathrm{~h}$ after disc excision, divided into two parts and frozen in liquid nitrogen for storage. All tissue samples were collected with written informed consent in accordance with the Declaration of Helsinki and 


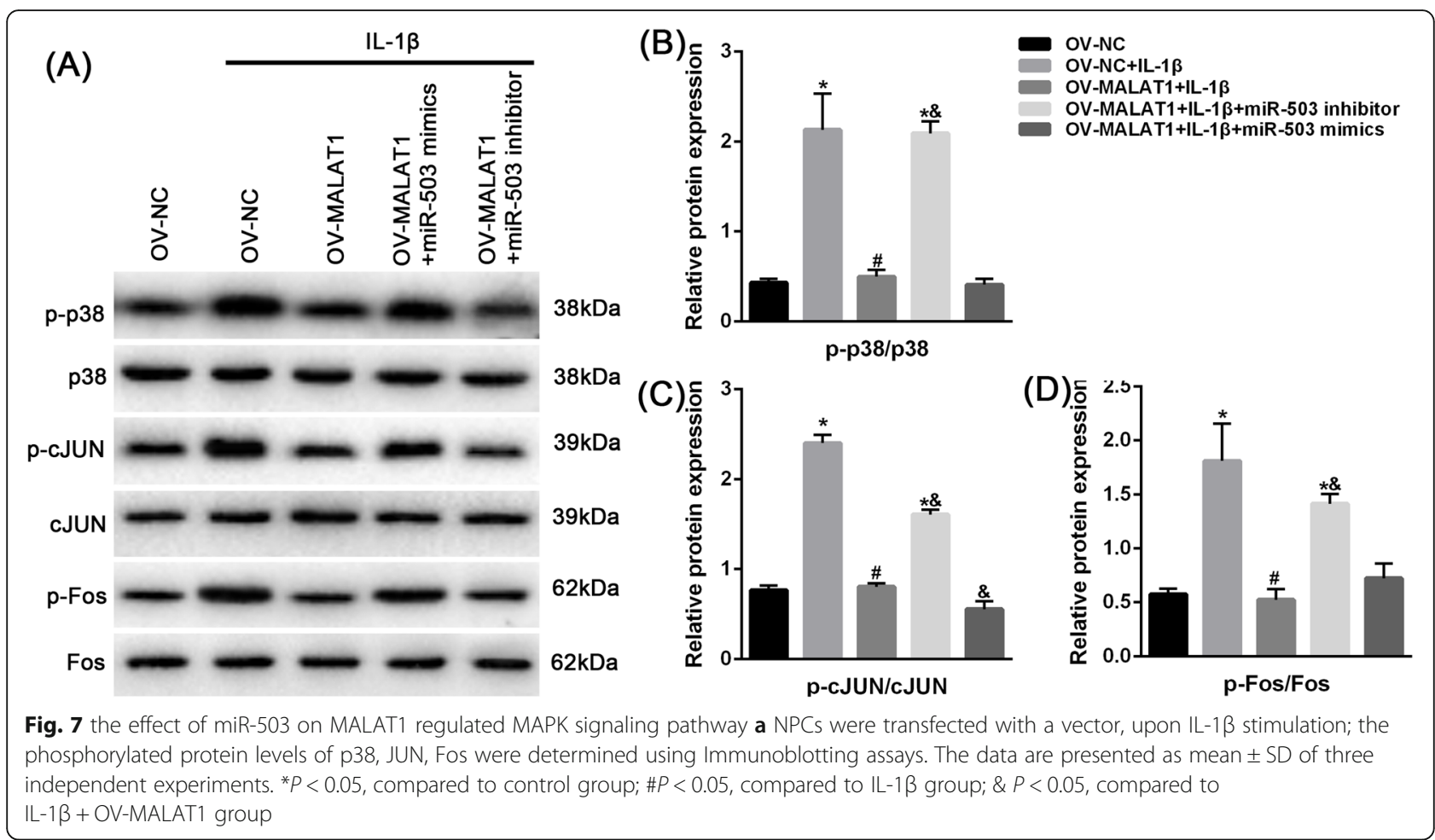

with the approval of the Medical Ethics Committee of the First Affiliated Hospital of Kunming Medical University (Approval number: 2018061811, Date: 2018/06/18, Kunming, China). All of the animal experiments in this study were performed in accordance with the National Institutes of Health Guide for Care and Use of Laboratory Animals, and were approved by the laboratory animal ethical committee of Kunming Medical University.

\section{NPC isolation and culture}

Normal human nucleus pulposus (HNP) tissues were gently separated from lumbar trauma patients under aseptic conditions, washed with D-Hank's solution 3-5 times, and then cut into small pieces with ophthalmic scissors $\left(<1 \mathrm{~mm}^{3}\right)$. Subsequently, tissues were placed overnight in $5 \mathrm{ml}$ of $0.1 \%$ type II collagenase (GIBCO, NY, USA) at $37^{\circ} \mathrm{C}$ for $8 \mathrm{~h}$. The digested fluid was filtered through 200 meshes filters, followed by filtration and centrifugation at $1000 \mathrm{rpm}$ for $5 \mathrm{~min}$. The supernatant was removed, and the precipitate was suspended in $3 \mathrm{ml}$ of medium and centrifuged at $1000 \mathrm{rpm}$ for $5 \mathrm{~min}$. The supernatant was removed again, and the NPCs were seeded into a culture flask in DMEM/F12 medium (GIBCO, NY, USA) containing $15 \%$ fetal bovine serum (FBS, GIBCO, NY, USA), $100 \mu \mathrm{g} / \mathrm{ml}$ streptomycin and $100 \mathrm{U} / \mathrm{ml}$ penicillin under $5 \% \mathrm{CO}_{2}$ and saturated humidity at $37^{\circ} \mathrm{C}$. The culture medium was changed three times a week, and NPCs were subcultured at a ratio of 1:3 after reaching $80 \%$ confluence.
Cell morphology was observed under an inverted microscope (DM6000B; Leica Microsystems, Japan). The concentration of HNPCs were adjusted to $1 \times$ $10^{4} / \mathrm{ml}$, and the cells were seeded into 24 -well plates with glass coverslips for $48 \mathrm{~h}$. Cells were fixed in formaldehyde for $20 \mathrm{~min}$, followed by three washes with phosphate-buffered saline (PBS).

\section{Plasmid construction, dual-luciferase assays, and cell transfections}

The lncRNA MALAT1 overexpression plasmid (OVMALAT1) and negative control (OV-NC) were purchased from GenePharma (Shanghai, China). The following sequence of siRNA oligonucleotides (si-MALAT1) was used to knockdown MALAT1 expression: 5' -CACAGGGAAA GCGAGUGGUUGGUA-3'. The sequence of the noncoding control siRNA (si-NC) was 5'-UUCUCCGAAC GUGUCACGU-3'. si-MALAT1, si-NC, mir-503 mimics, miR-503 inhibitor, and corresponding negative controls were purchased from RiboBio Co. (Guangzhou, China). Lipofectamine 2000 (Thermo Fisher Scientific, Waltham, USA) was used for transfection. Twenty-four hours before transfection, NP cells were seeded at $2 \times 10^{4}$ cells/well in a 96-well plate. Cells were transfected with OV-NC or OVMALAT1 using Lipofectamine 2000. Twenty-four hours after transfection, cells were treated with $150 \mathrm{ng} / \mathrm{mL} \mathrm{IL}-1 \beta$ for $6 \mathrm{~h}$. Forty-eight hours later, NPCs were used for the following experiments. 


\section{Luciferase assay}

To verify whether there is a director interaction between MALAT1 and miR-503, a pmirGLO Dual-Luciferase miRNA target expression vector was used for 3 '-untranslated region (UTR) luciferase assays (Promega, Madison, WI). $293 \mathrm{~T}$ cells were plated $\left(5 \times 10^{4}\right.$ cells per well) in 24-well plates and cells in each well were cotransfected with miR-503 and wild type or mutant target sequences using Lipofectamine 2000. Cells were then harvested $48 \mathrm{~h}$ after transfection, and the activities of firefly and Renilla luciferases were measured by using the Dual-Luciferase Reporter Assay System with the miR-NC set at 1.0. In addition, cells were seeded in 6well plates $\left(1 \times 10^{6}\right.$ cells/well, $2 \mathrm{ml}$ medium per well $)$ and cultured normally for $24 \mathrm{~h}$. Firefly luciferase activity was normalized to Renilla luciferase activity for each sample.

\section{RNA extraction and quantitative real-time PCR (qRT-PCR)} TRIzol reagent (Life Technologies, Gaithersburg, MD, USA) was used to extract total RNA from cells according to the manufacturer's instruction. Then, cDNA was synthesized using $1 \mu \mathrm{g}$ of total RNA as template and a RevertAidTM First Strand cDNA Synthesis Kit (Fermentas, Maryland, USA). Quantitative real-time PCR (qRT-PCR) analyses were performed with $\mathrm{SYBR}^{\bullet}$ Premix $\mathrm{Ex} \mathrm{Taq}^{\mathrm{Tm}}(\mathrm{Ta}$ kara, Japan) using a StepOne-Plus Real-Time PCR System (Applied Biosystems, USA). The PCR amplification included an initial denaturation at $95^{\circ} \mathrm{C}$ for $1 \mathrm{~min}, 35$ cycles of denaturation at $95^{\circ} \mathrm{C}$ for $1 \mathrm{~min}$, annealing at $60^{\circ} \mathrm{C}$ for $2 \mathrm{~min}$, and extension for $30 \mathrm{~s}$, at $72^{\circ} \mathrm{C}$. Results of the loglinear phase of the growth curve were analyzed, and relative quantification was performed using the $2^{-\Delta \Delta C T}$ method with GAPDH serving as a housekeeping gene. U6 snRNA was used as an internal control to normalize the expression levels of miRNAs. The PCR primers used were as follows: MALAT1, 5' - GACGGAGGTTGAGATGAA GC-3' and 5' - ATTCGGGGCTCTGTAGTCCT-3'; IL$1 \beta, 5^{\prime}$ - GACGGAGGTTGAGATGAAGC-3' and 5' - AT TCGGGGCTCTGTAGTCCT-3'; Collagen II, 5'- GAC GGAGGTTGAGATGAAGC-3' and 5' - ATTCGGGGCT CTGTAGTCCT-3'; Aggrecan, 5' - GACGGAGGTTGAG ATGAAGC-3' and 5' - ATTCGGGGCTCTGTAGTCCT3'; GAPDH, 5'-GAAGGTGAAGGTCGGAGTC-3' and 5'-GAAGATGGTGATGGGATTTC-3'.

\section{NPC proliferation by CCK-8}

The viability of HNPCs was evaluated using Cell Counting Kit-8 (Dojindo, Kumamoto, Japan), according to the manufacturer's instructions. In brief, cells were seeded in 96-well plates, and cultured for $8,16,24 \mathrm{~h}$ and stimulated with 150 $\mathrm{ng} / \mathrm{mL}$ IL-1 $\beta$ (GIBCO, NY, USA) for 8,16 , and $24 \mathrm{~h}$, respectively. NPCs were incubated with CCK- 8 reagent for 1 $\mathrm{h}$, and absorbance at $450 \mathrm{~nm}$ was measured with a microplate reader (Tecan, Männedorf, Switzerland).

\section{NPC apoptosis by Annexin V/propidium iodide (PI)} staining

Cell apoptosis was detected using the Annexin V-FITC/ PI staining method. After the cells were cultured for 24 $\mathrm{h}$ and stimulated with different concentrations of IL-1 $\beta$ $(150 \mathrm{ng} / \mathrm{mL})$ for the same culture time, they were washed twice with precooled PBS and digested with $0.25 \%$ trypsin, and the density was adjusted with DMEM to $1 \times 10^{6}$ cells $/ \mathrm{mL}$. After fixing with precooled $70 \%$ alcohol for $24 \mathrm{~h}$, the cells were centrifuged at $1000 \mathrm{r} / \mathrm{min}$ for $5 \mathrm{~min}$, followed by two washes with PBS. Next, $100 \mu \mathrm{L}$ of $1 \times 10^{6}$ cells $/ \mathrm{mL}$ cell suspension was added to $5 \mu \mathrm{L}$ of Annexin V-FITC (Dojindo, Kumamoto, Japan) and $10 \mu \mathrm{L}$ of PI, and the culture was incubated at room temperature for $15 \mathrm{~min}$ and washed twice with PBS. Cell apoptosis was analyzed using FACS Calibur Flow Cytometer (BD, USA). Each group had three replicates, and each process was repeated three times.

\section{Immunofluorescent staining}

NPCs were plated on coverslips in 6-well plates at $2 \times 10^{5}$ cells/well for $48 \mathrm{~h}$. After cyclic stretching, the cells were harvested for fluorescence labeling of Collagen II. Briefly, cells were washed twice with PBS and fixed with $4 \%$ paraformaldehyde for $15 \mathrm{~min}$. Then, after incubation with PBS containing $0.2 \%$ Triton $\mathrm{X}-100$ for $10 \mathrm{~min}$, cells were treated with PBS containing $5 \%$ bovine serum albumin (BSA) for $20 \mathrm{~min}$ to block nonspecific protein binding. After blocking, the cells were incubated overnight at $4{ }^{\circ} \mathrm{C}$ with primary antibodies (anti-Collagen II, 1:100, Santa Cruz) and then incubated with fluorescent secondary antibodies (1:200, Santa Cruz) at room temperature for $2 \mathrm{~h}$. The staining results were visualized under a fluorescence microscope in the same field.

\section{Protein extraction and wWestern blot analysis}

Total protein was extracted, and the protein concentration was quantified using a BCA protein assay kit (Beyotime Biotechnology, Jiangsu, China). A total of $20 \mu \mathrm{g}$ of protein from each sample was used for Western blotting. The samples were separated by SDS-PAGE(10\%) at $200 \mathrm{~V}$ for $50 \mathrm{~min}$. After transferring the proteins onto polyvinylidene fluoride (PVDF) membranes, the blotting was performed at $300 \mathrm{~mA}$ for $45 \mathrm{~min}$. After blocking with $5 \%(\mathrm{w} / \mathrm{v})$ dry milk in TBS for $1 \mathrm{~h}$ at room temperature. Membranes were incubated with the primary antibodies. The primary antibodies used in this study included monoclonal antiCollagen II (1:1000), Aggrecan (1:500) (Cell Signaling Technology, Beverly, MA), Fos (1:500), p38 (1:500), JNK (1:500) and their phosphorylated antibodies (1:500, Santa Cruz, CA, USA) and anti-GAPDH polyclonal antibody (1: 2000 Santa Cruz, CA, USA) at $4{ }^{\circ} \mathrm{C}$ overnight. Then the membranes were incubated with HRP-conjugated antirabbit or anti-mouse antibody (1:10000, Cell Signaling 
Technology, Beverly, MA) for $2 \mathrm{~h}$ at room temperature. Finally, the blots were developed with an enhanced chemiluminescence kit (Millipore, Billerica, MA, USA), and the bands were quantified densitometrically using a Bio-Rad imaging system (Hercules, CA). GAPDH was used as the loading control.

\section{Establishment of the rat IVD model}

Thirty-two male male Sprague-Dawley rats $(350 \mathrm{~g}$, aged three months) were obtained from Changsha Tian Qin Biotechnology Co., Ltd. (Changsha, Hunan, China). Rats were used for the experiments in vivo. Thirty-two rats were randomly divided into four groups: normal group (Control; $n=$ 8), IVD model group without treatment (NT; $\mathrm{n}=8$ ), IVD model with the treatment of $\mathrm{NC}$-overexpressing lentivirus (OV-NC; $\mathrm{n}=8$ ), and IVD model with the treatment of MALAT1- overexpressing lentivirus (OV-MALAT1; $\mathrm{n}=8$ ). All procedures were performed according to the National Institutes of Health Guide for Care and Use of Laboratory Animals and approved by the laboratory animal ethics committee of Kunming Medical University.

The rat model of IVD was built by annulus fibrosus (AF) needle puncture. In brief, general anesthesia was administered using $3.6 \%$ chloral hydrate and $10 \mathrm{~mL} / \mathrm{kg}$ intraperitoneal. After successful anesthesia, SD rats were placed supine on the operation table, the limbs were fixed, and the tail was disinfected. According to the tail body surface markers of the rat, the tail bones $\mathrm{C} 5$ and $\mathrm{C} 6$ were determined. The middle part of the vertebral body was tilted by $45^{\circ}$ to the left and right, and two diameters of $0.8 \mathrm{~mm}$ Kirschner wire were drilled. The carbon fiber loops were successively fixed on the crossed Kirschner wires, and the carbon fiber rods and springs were inserted. The experimental group was given a spring nut to fix the pressure, and the four springs were compressed to $10 \mathrm{~mm} / \mathrm{kg}$ body weight (simulating the weight of the lumbar spine when the rat was walking upright). The compression length of the spring was controlled by a Vernier caliper; the control group did not pressurize the spring. After the operation, the two groups of animals were kept in a single cage, and penicillin 200,000 U was intramuscularly injected for three days to prevent infection.

$\mathrm{SD}$ rats were anesthetized with isoflurane gas (under a small animal anesthesia instrument). After anesthesia, the rats were placed on the operating table to fix the limbs. Under the guidance of fluoroscopy, in the three positions C6-C7, C8-C9, C10-C11, a 20 gauge needle was used to puncture the dorsal side, the puncture needle passed through the center of the intervertebral disc, until the opposite side, rotated $180^{\circ}$, and held for $10 \mathrm{~s}$. The wound was wrapped with gauze after surgery and anti-infective treatment was carried out. One week after surgery, the rats were anesthetized again, and a small incision was made to the left of the puncture site to expose the position of the previous puncture. The intervertebral disc was pierced with a 33 gauge needle, followed by injection of OV-MALAT1 lentivirus or OV-NC lentivirus, and injections were repeated four weeks later.

\section{X-ray and MRI examination}

At four weeks after injection, X-ray and MRI examinations were performed on all rats in the study. After examinations, five rats in each group were sacrificed by intraperitoneal injection of sodium pentobarbital. The tissues at the corresponding sites were harvested, fixed in $4 \%$ paraformaldehyde, and decalcified with 10\% EDTA solution. The treated tissue was embedded in paraffin and sectioned in the sagittal plane. The disc was collected and stored at $-80^{\circ} \mathrm{C}$ for further expression analysis.

\section{Histomorphology and TUNEL assay of the lumbar spine}

After the MRI examination, the rats were killed by intraperitoneal administration of overdose pentobarbital sodium. Lumbar spines including L4-L6 levels were harvested en bloc and then fixed in 4\% paraformaldehyde for $48 \mathrm{~h}$, decalcified at $4{ }^{\circ} \mathrm{C}$ in $20 \%$ ethylenediamine tetraacetic acid for 5-7 weeks, embedded in paraffin, and sectioned $(4 \mu \mathrm{m})$ along the midsagittal plane. Sections were used for hematoxylin-eosin (HE) or terminal deoxynucleotidyl transferase (TdT)-mediated dUTP nick end labeling (TUNEL). IDD was scored based on histomorphological features of HE-stained sections according to the classification system [28]. The average scores of L4-L5 and L5-L6 were recorded as the grade of lumbar IDD in each rat. Total and TUNEL-positive disc cells were counted below three to five noncontinuous high-power fields (magnification, $\times 400$ ) in each of the two regions (outer or inner AF) from each of two discs per specimen and summed up. The percentage of TUNEL- positive disc cells compared with total disc cells was then calculated.

\section{Statistical analysis}

Statistical differences between two groups were analyzed using Student's t-test. Differences among three or more groups were analyzed using one-way analysis of variance and Tukey's post hottest test. Data analysis was performed with GraphPad Prism 5 (Graphpad Software, La Jolla, CA, USA) and presented as the means $\pm \mathrm{SD}$. The difference was considered to be significant when $p<0.05$.

\section{Supplementary information}

Supplementary information accompanies this paper at https://doi.org/10. 1186/s12860-020-00265-2.

Additional file 1: Table S1 Significant pathways on putative target genes (3-UTR region) of miR-503.

\section{Abbreviations}

IDD: Intervertebral disc degeneration; NPCs: Nucleus pulposus cells; IncRNAs: Long non coding RNAs; ceRNA: Competing endogenous RNA; 
MALAT1: Metastasis-associated lung adenocarcinoma transcript-1; miRs: microRNAs; MRI: magnetic resonance imaging; HNP: Human nucleus pulposus; DMEM: Dulbecco's modified Eagle's medium; FBS: Fetal bovine serum; PI: Propidium iodide; IL-1ß: Interleukin 1 beta; UTR: Untranslated region; SDS-PAGE: Sodium dodecyl sulfate polyacrylamide gel electrophoresis; PVDF: Polyvinylidene fluoride membranes; TUNEL: Terminal deoxynucleotidyl transferase (TdT)-mediated dUTP nick end labeling

\section{Acknowledgments}

The authors thank Bo Yang for processing the data and drawing the graphs.

\section{Authors' contributions}

$\mathrm{HZ}$ and TW analyzed data and wrote the original draft. XL, WH, ZG and ZL performed and analyzed the experiments in this study. BW and $\mathrm{XL}$ conceived and coordinated the study. XL reviewed and edited the paper. All authors read and approved the final manuscript.

\section{Funding}

This work was supported by National Natural Science Foundation of China (Grant 81760029 and 81760030); Joint Project on Applied Basic Research Foundation of Yunnan Science and Technology Department-Kunming Medical University (Grant 2015FB030; 2018FE001-146 and 2018FE001-213); Yunnan Health Science and Technology Project (Grant 2017NS022); Kunming Medical University young and middle-aged academic and technical talents fund (Grant 60117190455); Kunming Health and Family Planning Commission Medical and Health Science and Technology Program - Reserve Talents (Thousand Project) (Grant 2019-03-07-003); and Scientific and Technological Innovation Team of Spinal Degenerative Diseases Research in Kunming Medical University (Grant CXTD201805). The funders had no role in study design, data collection and analysis, decision to publish, or preparation of the manuscript.

\section{Availability of data and materials}

The datasets supporting the conclusions of this article are included within the article.

\section{Ethics approval and consent to participate}

All tissue samples were collected with written informed consent in accordance with the Declaration of Helsinki and with the approval of the Medical Ethics Committee of the First Affiliated Hospital of Kunming Medical University (Approval number: 2018061811, Date: 2018/06/18, Kunming, China). All of the animal experiments in this study were performed in accordance with the National Institutes of Health Guide for Care and Use of Laboratory Animals, and were approved by the laboratory animal ethical committee of Kunming Medical University.

\section{Consent for publication}

Not applicable.

\section{Competing interests}

The authors declare that they have no competing interests.

\section{Author details}

'Department of Emergency Medical, First Affiliated Hospital of Kunming Medical University, Kunming, China. 'Department of Geriatrics, Yan' An Hospital of Kunming City, Kunming, China. ${ }^{3}$ Department of Panicaceae, First Affiliated Hospital of Kunming Medical University, Kunming, China. ${ }^{4}$ Department of Orthopedics, Qianjiang Central Hospital, Qianjiang, China. ${ }^{5}$ Department of Orthopedics, First Affiliated Hospital of Kunming Medical University, No. 295 Xichang Road, Kunming, Yunnan, China.

\section{Received: 12 November 2019 Accepted: 18 March 2020}

\section{Published online: 30 March 2020}

\section{References}

1. Fontana G, See E, Pandit A. Current trends in biologics delivery to restore intervertebral disc anabolism. Adv Drug Deliv Rev. 2015;84:146-58..

2. Pattappa G, Li Z, Peroglio M, Wismer N, Alini M, Grad S. Diversity of intervertebral disc cells: phenotype and function. J Anat. 2012;221(6):480-96

3. Arkesteijn ITM, Potier $E_{\text {I I }}$ Io K. The regenerative potential of Notochordal cells in a nucleus Pulposus explant. Global Spine J. 2017;7(1):14-20.
4. Wang X, Wang B, Zou M, Li J, Lu G, Zhang Q, Liu F, Lu C. CircSEMA4B targets miR-431 modulating IL-1beta-induced degradative changes in nucleus pulposus cells in intervertebral disc degeneration via Wnt pathway. Biochim Biophys Acta Mol basis Dis. 2018;1864(11):3754-68.

5. Cheng X, Zhang G, Zhang L, Hu Y, Zhang K, Sun X, Zhao C, Li H, Li YM, Zhao J. Mesenchymal stem cells deliver exogenous miR-21 via exosomes to inhibit nucleus pulposus cell apoptosis and reduce intervertebral disc degeneration. J Cell Mol Med. 2018;22(1):261-76.

6. Ding F, Shao ZW, Xiong LM. Cell death in intervertebral disc degeneration. Apoptosis. 2013;18(7):777-85.

7. Martirosyan NL, Patel AA, Carotenuto A, Kalani MY, Belykh E, Walker CT, Preul MC, Theodore N. Genetic alterations in intervertebral disc disease. Front Surg. 2016;3:59.

8. Mercer TR, Dinger ME, Mattick JS. Long non-coding RNAs: insights into functions. Nat Rev Genet. 2009:10(3):155.

9. Yu Y, Zhang X, Li Z, Kong L, Huang Y. LncRNA HOTAIR suppresses TNF-a induced apoptosis of nucleus pulposus cells by regulating miR-34a/Bcl-2 axis. Biomed Pharmacother. 2018;107:729-37.

10. Guo C, Wang X, Chen L, Li M, Li M, Hu Y, Ding W, Wang X. Long noncoding RNA MALAT1 regulates ovarian cancer cell proliferation, migration and apoptosis through Wnt/beta-catenin signaling pathway. Eur Rev Med Pharmacol Sci. 2018;22(12):3703-12.

11. Zhou H-J, Wang L-Q, Wang D-B, Yu J-B, Zhu Y, Xu Q-S, Zheng X-J, Zhan R-Y. Long noncoding RNA MALAT1 contributes to inflammatory response of microglia following spinal cord injury via the modulation of a miR-199b/IKKß/NF-KB signaling pathway. Am J Phys Cell Phys. 2018; 315(1):C52-61.

12. Yan W, Wu Q, Yao W, Li Y, Liu Y, Yuan J, Han R, Yang J, Ji X, Ni C. MiR-503 modulates epithelial-mesenchymal transition in silica-induced pulmonary fibrosis by targeting PI3K p85 and is sponged by IncRNA MALAT1. Sci Rep. 2017;7(1):11313

13. Li Q, Li C, Xi S, Li X, Ding L, Li M. The effects of photobiomodulation therapy on mouse pre-osteoblast cell line MC3T3-E1 proliferation and apoptosis via miR-503/Wnt3a pathway. Lasers Med Sci. 2019:34(3):607-14.

14. Wang J, Hu J, Chen X, Huang C, Lin J, Shao Z, Gu M, Wu Y, Tian N, Gao W. BRD4 inhibition regulates MAPK, NF-KB signals, and autophagy to suppress MMP-13 expression in diabetic intervertebral disc degeneration. FASEB J. 2019:33(10):11555-66.

15. J-k L, Nie L, Zhao Y-P, Zhang Y-Q, Wang X, Wang S-S, Liu Y, Zhao H, Cheng L. IL-17 mediates inflammatory reactions via p38/c-Fos and JNK/c-Jun activation in an AP-1-dependent manner in human nucleus pulposus cells. J Transl Med. 2016;14(1):77.

16. Studer RK, Aboka AM, Gilbertson LG, Georgescu H, Sowa G, Vo N, Kang JD. p38 MAPK inhibition in nucleus pulposus cells: a potential target for treating intervertebral disc degeneration. Spine. 2007;32(25):2827-33.

17. Roberts S, Caterson B, Menage J, Evans EH, Jaffray DC, Eisenstein SM. Matrix metalloproteinases and aggrecanase: their role in disorders of the human intervertebral disc. Spine. 2000;25(23):3005-13.

18. Wuertz K, Vo N, Kletsas D, Boos N. Inflammatory and catabolic signalling in intervertebral discs: the roles of NF-KB and MAP kinases. Eur Cell Mater. 2012;23:103-19.

19. Tasharrofi B, Ghafouri-Fard S. Long non-coding RNAs as regulators of the mitogen-activated protein kinase (MAPK) pathway in cancer. Klin Onkol. 2018;31:95-102.

20. Le Maitre CL, Freemont AJ, Hoyland JA. The role of interleukin-1 in the pathogenesis of human intervertebral disc degeneration. Arthritis Res Ther. 2005;7(4):R732-45.

21. Le Maitre CL, Hoyland JA, Freemont AJ. Catabolic cytokine expression in degenerate and herniated human intervertebral discs: IL-1ß and TNFa expression profile. Arthritis Res Ther. 2007;9(4):R77.

22. Yamamoto J, Maeno K, Takada T, Kakutani K, Yurube T, Zhang Z, Hirata H, Kurakawa T, Sakai D, Mochida J. Fas ligand plays an important role for the production of pro-inflammatory cytokines in intervertebral disc nucleus pulposus cells. J Orthop Res. 2013:31(4):608-15.

23. Rand N, Reichert F, Floman Y, Rotshenker S. Murine nucleus pulposusderived cells secrete interleukins-1- $\beta,-6$, and-10 and granulocytemacrophage colony-stimulating factor in cell culture. Spine. 1997;22(22): 2598-601.

24. Kepler CK, Markova DZ, Hilibrand AS, Vaccaro AR, Risbud MV, Albert TJ, Anderson DG. Substance $P$ stimulates production of inflammatory cytokines in human disc cells. Spine. 2013;38(21):E1291-9. 
25. Zhang H, Li J, Duan D, She W, Wang L, Zhang F. The role of IncRNA MALAT1 in intervertebral degenerative disc disease. Int J Clin Exp Pathol. 2017;10(10):10611-7.

26. Liu Z, Ma C, Shen J, Wang D, Hao J, Hu Z. SDF-1/CXCR4 axis induces apoptosis of human degenerative nucleus pulposus cells via the NF-KB pathway. Mol Med Rep. 2016;14(1):783-9.

27. Wang Y-J, Shi Q, Lu WW, Cheung KC, Darowish M, Li T-F, Dong Y-F, Zhou CJ, Zhou Q. Hu Z-j: cervical intervertebral disc degeneration induced by unbalanced dynamic and static forces: a novel in vivo rat model. Spine. 2006;31(14):1532-8.

28. Chen C, Cheng P, Xie H, Zhou HD, Wu XP, Liao EY, Luo XH. MiR-503 regulates osteoclastogenesis via targeting RANK. J Bone Miner Res. 2014; 29(2):338-47.

29. M-I J, X-j Z, P-I S, Lu J, Wang S-Z, Chang Q, Chen H, Wang C. Downregulation of microRNA-193a-3p is involved in invertebral disc degeneration by targeting MMP14. J Mol Med. 2016;94(4):457-68.

30. H-r L, Cui Q, Dong Z-Y, Zhang J-H, Li H-Q, Zhao L. Downregulation of miR$27 \mathrm{~b}$ is involved in loss of type II collagen by directly targeting matrix metalloproteinase 13 (MMP13) in human intervertebral disc degeneration. Spine. 2016;41(3):E116-23.

31. Yokoyama K, Hiyama A, Arai F, Nukaga T, Sakai D, Mochida J. C-Fos regulation by the MAPK and PKC pathways in intervertebral disc cells. PLoS One. 2013;8(9):e73210.

32. Wagner EF, Nebreda ÁR. Signal integration by JNK and p38 MAPK pathways in cancer development. Nat Rev Cancer. 2009;9(8):537.

33. Daniels J, Binch AA, Le Maitre CL. Inhibiting IL-1 signaling pathways to inhibit catabolic processes in disc degeneration. J Orthop Res. 2017;35(1):74-85.

34. Ulrich JA, Liebenberg EC, Thuillier DU, Lotz JC. ISSLS prize winner: repeated disc injury causes persistent inflammation. Spine. 2007;32(25):2812-9.

35. Li Y, Liu Y-D, Chen S-L, Chen X, Ye D-S, Zhou X-Y, Zhe J, Zhang J. Downregulation of long non-coding RNA MALAT1 inhibits granulosa cell proliferation in endometriosis by up-regulating P21 via activation of the ERK/MAPK pathway. MHR: Basic Sci Reprod Med. 2019;25(1):17-29.

36. Chen $H$, Wang $X$, Yan $X$, Cheng $X$, He $X$, Zheng W. LncRNA MALAT1 regulates sepsis-induced cardiac inflammation and dysfunction via interaction with miR-125b and p38 MAPK/NFkB. Int Immunopharmacol. 2018;55:69-76

37. Zou A, Liu R, Wu X. Long non-coding RNA MALAT1 is up-regulated in ovarian cancer tissue and promotes SK-OV-3 cell proliferation and invasion. Neoplasma. 2016;63(6):865-72.

38. Jones TA, Jeyapalan JN, Forshew $T$, Tatevossian RG, Lawson AR, Patel SN, Doctor GT, Mumin MA, Picker SR, Phipps KP. Molecular analysis of pediatric brain tumors identifies microRNAs in pilocytic astrocytomas that target the MAPK and NF-KB pathways. Acta Neuropathol Commun. 2015;3(1):86.

39. Sticht C, De La Torre C, Parveen A, Gretz N. miRWalk: An online resource for prediction of microRNA binding sites. PLoS ONE. 2018;13(10):e0206239.

\section{Publisher's Note}

Springer Nature remains neutral with regard to jurisdictional claims in published maps and institutional affiliations.

Ready to submit your research? Choose BMC and benefit from:

- fast, convenient online submission

- thorough peer review by experienced researchers in your field

- rapid publication on acceptance

- support for research data, including large and complex data types

- gold Open Access which fosters wider collaboration and increased citations

- maximum visibility for your research: over $100 \mathrm{M}$ website views per year

At $\mathrm{BMC}$, research is always in progress.

Learn more biomedcentral.com/submissions 